\title{
Enhancement of Computer Network Performance with VLAN
}

\author{
Achmad Ubaidillah $^{1 *}$, Koko Joni ${ }^{1}$, Mohammad Iqbal Bachtiar ${ }^{1}$ and S. Ida Kholida ${ }^{2}$ \\ ${ }^{1}$ Electrical Engineering, Trunojoyo Madura University, Bangkalan, East Java, Indonesia \\ ${ }^{2}$ Physics Education, Madura Islamic University, Pamekasan, East Java, Indonesia
}

\begin{abstract}
The computer network service provider must provide a good network service. Service quality is the most important factor and must always be maintained properly. The quality of service can change depending on the number and characteristics of service users. This study optimizes the performance of existing computer networks, namely ordinary LAN networks with the application of VLANs. It is very important because the number of network users is increasing and will continue to increase, while the need for good service quality is very urgent. The results showed that VLANs can improve computer network performance from the system existing, namely LAN network.
\end{abstract}

Keyword: Service Quality, LAN, Computer Network Performance

\section{Introduction}

A local Area Network (LAN) is a group of computers connected together in a small geographic area to communicate with each other via wired or wireless links and share resources such as printers and network storage. Virtual Local Area Network (VLAN) is a virtual activated LAN network. Theoretically, some deficiencies in LAN networks can be overcome by VLANs [1]. In a VLAN implementation, workstations, servers and other peripheral devices used by certain work groups which are geographically dispersed are grouped and can communicate with each other as if they were physically in the same location in the network. VLAN networks are designed to break up large broadcast domains into smaller ones. One VLAN switch network device can have many broadcast domains [2].

Research on the application of VLANs has been carried out a lot. Some of them are the comparison of LAN and VLAN performance using Cisco and Brocade [3]. Another study evaluated the application of VLANs and its effect on the performance of computer networks with different generator traffic [4]. Even the development of VLAN implementation is carried out to an effective routing system [5].

Internet network quality monitoring is carried out by network administrators. Some of the metrics commonly used in measuring network quality are throughput, delay, jitter, and packet loss. The quality of internet access is greatly influenced by the amount of bandwidth and the number of users. Optimization is required for management of limited network resources [6].

Quality of Service (QOS) measurement is the most important in communication network installations. A communication network system is categorized as good or bad quality depending on its QoS. Thus, almost all research in the field of telecommunications networks ends at QoS measurements. For example, QoS is used to measure the quality of routing protocol development [7], mobile cloud computing development [8], even in the application of a method to optimize the performance of a telecommunications network system [9].

In this study, the application of VLANs will be observed and analyzed whether it can optimize computer network performance or not, compared to ordinary LAN networks.

This section discusses the introduction and literature review of the research. The second part discusses the general theory of VLANs and Quality of Service. The third section discusses the research methodology. The fourth section discusses the research results and discussion. And the last section is the fifth section, namely conclusions and suggestions.

\section{Literature Review}

VLAN is applied with a unique identity called VLAN ID. VLAN ID can be found based on the address of the Media Access Control (MAC) Address. Some of the advantages of VLANs are [10]:

- VLANs are able to reduce the amount of unnecessary data sent to the destination, thereby reducing the network load.

- VLANs can make it easier for network administrators to work. Every time a computer moves, it must be reconfigured to be able to communicate with the network in the new area. This made the computer inoperable immediately after being moved. A network with the VLAN

* Corresponding author : ubaidillah.ms@trunojoyo.ac.id 
Principle can minimize or even remove this step because basically it remains on the same network.

- Moving locations on a normal LAN network will incur additional installation costs. VLANs can minimize these additional costs and even delete them.

- In terms of network security, VLANs can limit users who can access data, thereby reducing the possibility of misuse of access.

QoS is a technique for managing bandwidth, throughput, delay, jitter, and packet loss in order to measure the performance of telecommunications networks [11]. QoS is designed to help end users or clients to be more productive by ensuring that users get reliable performance from network-based applications. QoS refers to a network's ability to provide better service to specific network traffic through different technologies. Another goal of QoS is to meet different service requirements, using the same infrastructure. QoS offers the ability to define the attributes of the services provided, both qualitatively and quantitatively [12].

The several metrics in network QoS measurement are:

Delay. Delay is the time required for transmission from source to destination.

Throughput. Throughput is the total number of successfully received packets observed at the destination during a certain time interval.

Jitter. Jitter is a variation of the delay. Jitter is influenced by variations in traffic load and the amount of congestion between packets in the network. The effect of jitter on network performance must be seen in conjunction with delay. When the jitter is great but the delay is small, so the network performance cannot be said to be bad because the large amount of jitter can be compensated for by a small delay value.

Packet Loss. Packet loss is the number of packets that fail to reach their destination. When the packet lost is large, it means that the network load is high.

\section{Methodology}

The steps in this research are described in the following flow chart.

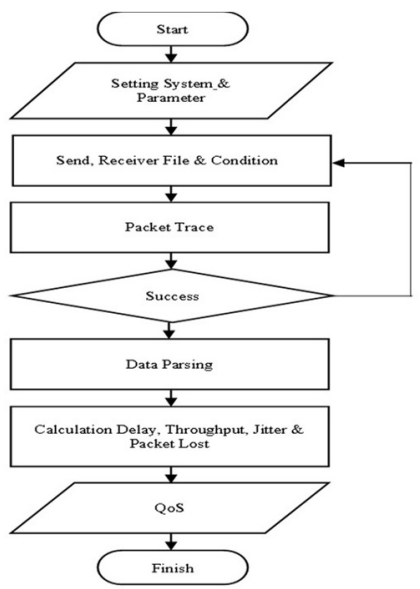

Fig. 1. The flow chart of research steps.
The tested topology is as shown in Fig. 2 below.

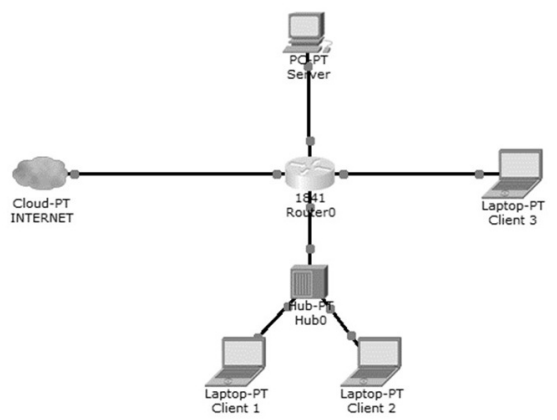

Fig. 2. Flow chart of research steps.

\section{Results and Discussion}

The value of throughput, delay, jitter and packet loss from the test results with variations in the size of the uploaded packets and it's comparison between LAN and VLAN networks are shown in Fig. 3, Fig. 4, Fig. 5 and Fig. 6.

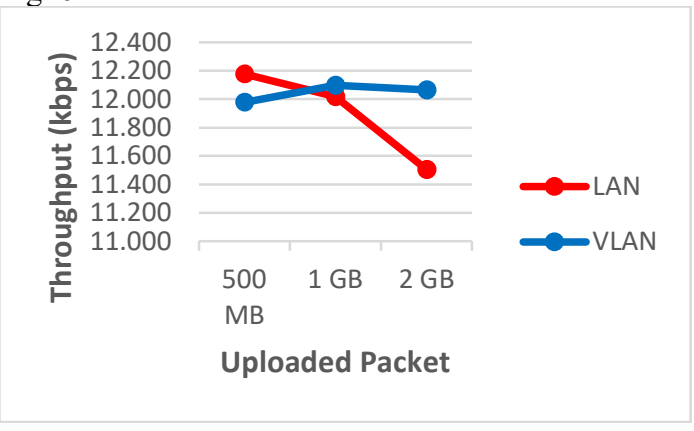

Fig. 3. Comparison of throughput on LAN and VLAN networks with variations in the size of uploaded packets

From Fig. 3, it can be seen that the throughput on the VLAN network is better than on the LAN. Throughput on the LAN network decreases when the traffic load is full, which is manifested by the enlarged uploaded packet size. Meanwhile, in VLAN networks, throughput tends to be stable.

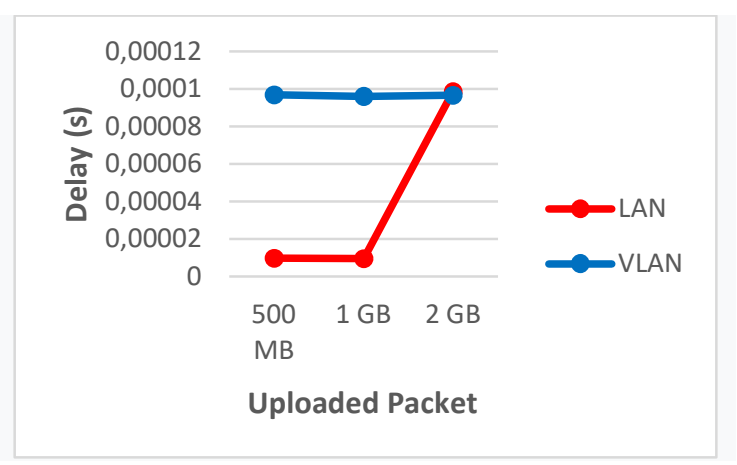

Fig. 4. Comparison of delay on LAN and VLAN networks with variations in the size of uploaded packets 
Like throughput analysis, delay on VLAN network is better than LAN. As the traffic load increases, the delay on the LAN network gets worse or the delay value gets higher. While in VLAN network, delay tends to be stable.

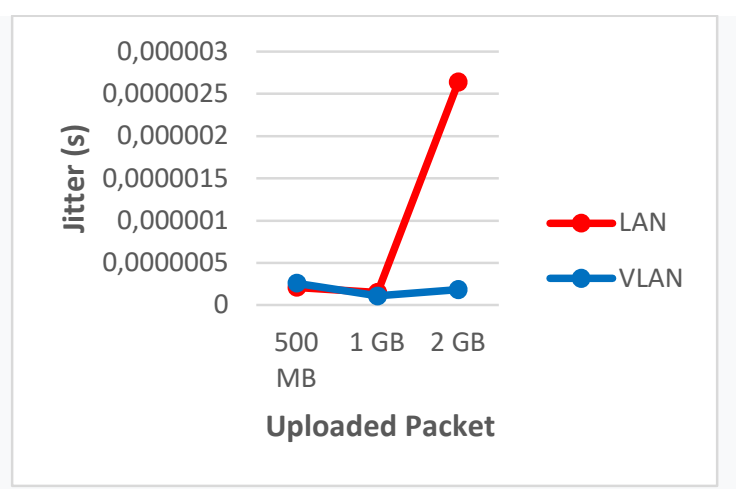

Fig. 5. Comparison of jitter on LAN and VLAN networks with variations in the size of uploaded packets

Fig. 5 can be analyzed that the jitter on the VLAN network is better than the LAN and it shows that the delay on the VLAN network is more stable than the LAN. The increasingly dense traffic load conditions on the LAN network cause a higher delay so that the jitter value becomes high.

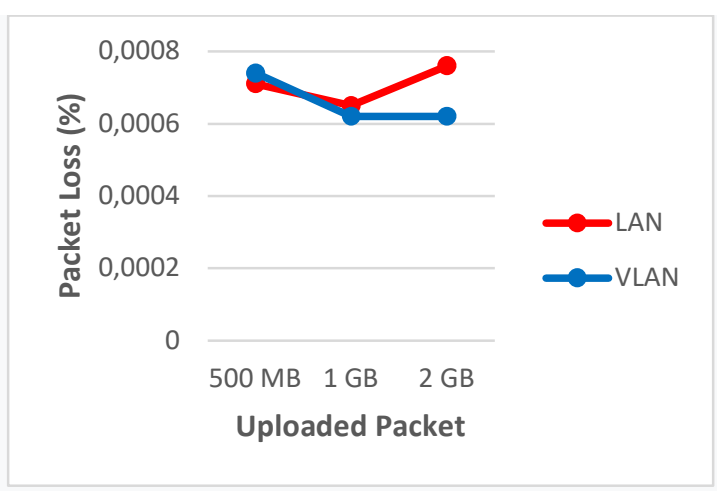

Fig. 6. Comparison of packet loss on LAN and VLAN networks with variations in the size of uploaded packets

Fig. 6 shows that packet loss in VLAN networks tends to be lower or better than LAN networks. This is because the options that have the potential to cause packet loss on the LAN network such as the topology hierarchy can be simplified in the VLAN network.

\section{Conclusion}

Some conclusions from this research are that the VLAN network is more stable than LAN, namely the value of throughput, delay, jitter and packet loss. VLAN network is more reliable than LAN in high traffic load conditions, namely with higher throughput value, lower delay value, lower jitter and lower packet loss. So it can be said that VLANs can improve the performance of ordinary computer networks with LANs.

Suggestions for further research is to conduct research with large networks or real networks, measured under conditions of low, medium and high traffic loads, to determine the behavior of a more comprehensive VLAN network system. It is also recommended to do research to find out under which conditions the LAN network becomes more optimal and under which conditions the VLAN network becomes more optimal.

\section{References}

[1] A. I. Mathew and S. R. B. Prabhu, "A Study on Virtual Local Area Network (Vlan) and InterVlan Routing," Int. J. Curr. Eng. Sci. Res., vol. 4, no. 10, pp. 2393-2395, (2017), [Online]. Available: https://papers.ssrn.com/abstract=3055382.

[2] M. S. Bahry and B. Sugiantoro, "Analysys and Implementation IEEE 802.1Q to Improve Network Security," IJID (International J. Informatics Dev., vol. 6, no. 2, p. 7, (2018), doi: 10.14421/ijid.2017.06202.

[3] R. Gatra, R. Akbar, B. Sugiantoro, and N. Asyhab, "VLAN-based LAN Network Management Comparison using Cisco and Brocade," IJID (International J. Informatics Dev., vol. 7, no. 2, p. 45, (2019), doi: 10.14421/ijid.2018.07208.

[4] Z. Nagy and M. K. Wali, "Virtual private network impacts on the computer network performance with different traffic generators," IOP Conf. Ser. Mater. Sci. Eng., vol. 881, no. 1, (2020), doi: 10.1088/1757-899X/881/1/012126.

[5] C. O. Agwu, N. E. Nwogbaga, and C. N. Ojiugwo, "The Proposed Roles of VLAN and Inter-VLAN Routing in Effective Distribution of Network Services in Ebonyi State University," Int. J. Sci. Res., vol. 4, no. 7, pp. 2608-2615, (2013).

[6] G. A. Qadir and S. R. M. Zeebaree, "Evaluation of QoS in Distributed Systems : A Review," Int. J. Sci. Bus., vol. 5, no. 2, pp. 89-101, (2021), doi: 10.5281 /zenodo.4481463.

[7] S. Mostafavi, V. Hakami, and F. Paydar, "INTERNATIONAL JOURNAL ON INFORMATICS VISUALIZATION A QoSAssured and Mobility-Aware Routing Protocol for MANETs," vol. 4, pp. 1-9.

[8] C. Denninnart, "Cost- and QoS-Efficient Serverless Cloud Computing," 2020, [Online]. Available: http://arxiv.org/abs/2011.11711.

[9] S. Saraireh and M. Saraireh, "Fuzzy Based System For Assessing And Enhancing Qos For Wireless Networks," vol. 9, no. 09, pp. 267273, (2020).

[10] D. E. Bassey, B. E. Okon, and R. Umunnah, "The Security Implications of Virtual Local Area Network (VLAN),Niger Mills, Calabar, Nigeria," Int. J. Sci. Eng. Res., vol. 7, no. 3, pp. 1187-1194, (2016). 
[11] L. F. Hussein, A. H. AbdallaHashim, M. H. Habaebi, and W. H. Hassan, "An adaptive diffserv approach to support QOS in network mobility NEMO environment," Int. J. Comput. Networks Commun., vol. 12, no. 2, pp. 23-48, (2020), doi: 10.5121/ijcnc.2020.12202.

[12] A. Ubaidillah and S. I. Kholida, "Forecasting of Computer Network Performance," vol. 03004, (2016). 